A0014: Perception of Epilepsy Stigma in School Children and Teachers' Awareness and Attitude towards Epilepsy-A South Indian Cohort Study

G.V.K.R. Goud, ${ }^{1}$ Balakrishna, ${ }^{1}$ Hemalatha, ${ }^{1}$ Rajeswari, ${ }^{1}$ Vasavi Chinta, ${ }^{1}$ Suryaprabha, ${ }^{1}$ Rukmini Mrudula, ${ }^{1}$ Rupam Borgohain $^{1}$

${ }^{1}$ Department of Neurology, Nizam's Institute of Medical Sciences, Hyderabad, Telangana, India

Background: School drop outs, due to stigma, due to epilepsy is a serious problem in India and school children and teachers play a dominant role as backbones for any awareness program. Understanding the attitudes and stigma toward epilepsy of school children and teachers may influence the society in the form of improving quality of life of people with epilepsy. There are not many studies addressing this aspect, especially in India.

Aim: and Objectives To study the perception of epilepsy stigma in school children and the awareness and attitude of teachers toward epilepsy in Singareni Collieries, an area of Telangana, in South India.

Materials and Methods: A total of 508 school children and 30 teachers of three schools were enrolled. The stigma scale of epilepsy (SSE) was applied to school children and a standardized questionnaire was applied to teachers to assess the awareness and attitude toward epilepsy.

Results: The overall mean score for epilepsy stigma perception was high being $54+2.5$. The SSE score for women (55.8) was higher than that for men (51.5). Only 25\% admitted are well informed about epilepsy. But 23\% respondents thought that epilepsy effected the education of a person.

Conclusion:s Perceived stigma was found to be a common problem in school children. Lack of awareness and negative attitude of teachers against people with epilepsy is likely to contribute to perceived feeling of stigma. The Results reinforce the need for creating awareness among school children and teachers addressing misconceptions attached to epilepsy.

\section{A0015: Depression in Persons with Epilepsy: A Comparative Study of Different Screening Tools in Indian Population \\ Haroon Rashid, ${ }^{1}$ Jatinder Katyal,, ${ }^{1}$ Manjari Tripathi, ${ }^{1}$ Mamta Sood, ${ }^{1}$ Yogendra K. Gupta ${ }^{1}$ \\ ${ }^{1}$ Neuropharmacology Laboratory, Department of Pharmacology, All India Institute of Medical Sciences, New Delhi, India.}

Objectives: Depression is a major comorbidity in persons with epilepsy ( $p W E$ ) and its timely identification is essential. In this study, depression in PWE was assessed and potential screening tools were compared head-to-head.

Methods: After ethical clearance, 217 PWE above 18 years of age, on antiepileptic drugs (AEDs), attending neurology outpatient department (OPD) at All India Institute of Medical Sciences (AIIMS), New Delhi, were evaluated for depression using four different screening tools head-to- head: MINI, NDDI-E, PHQ 9, and HAM-D scale. Informed consent was taken before recruitment.
Results: Of the 217 PWE (112 male/105 female) with a mean age of 28.6 years, $69.1 \%$ had a diagnosis of generalized and $30.9 \%$ focal seizures, majority (59\%) were on monotherapy, levetiracetam $(n=58)$, sodium valproate $(n=28)$, carbamazepine ( $n=26)$, phenytoin $(n=9)$, clobazam $(n=4)$, clonazepam $(n=1)$, oxcarbazepine $(n=1)$, and lamotrigine $(n=1)$, while those on polytherapy $(41 \%)$ received a combination of two to five of these AEDs. Using different scales, a variable percentage of PWE were screened positive for depression-41.5\% with MINI, 24\% with NDDI-E, 47.2\% with HAM-D, and 48.3\% with PHQ 9. When MINI scale was taken as reference standard, variable sensitivity and specificity of scales was found. At the recommended cut-off scores, HAM-D had $98 \%$ sensitivity and 91\% specificity, PHQ-9 showed $100 \%$ sensitivity and $89 \%$ specificity, and the NDDI-E showed sensitivity of $55.6 \%$ and specificity of $98.4 \%$.

Conclusion: A periodic assessment of PWE for depression is highly desirable. All the scales used in this study were found to be appropriate for use in PWE providing the cutoff points are properly validated as variation is possible.

\section{A0016: Association between Epilepsy and Headache: A Case Control Study Hemant Joshi, ${ }^{1}$ Chaturbhuj Rathore, ${ }^{1}$ Sanjay Prakash ${ }^{1}$ ${ }^{1}$ Department of Neurology, Smt. B. K. Shah Medical Institute and Research Centre, Vadodara, Gujarat, India}

Aim: To study the prevalence of headache in patients with epilepsy and their first degree relatives as compared with controls.

Methods: Consecutive patients, older than 5 years, with active epilepsy attending the epilepsy clinic and their first-degree relatives were interviewed for the presence and type of headaches. Patients with mental retardation and communication difficulties were excluded. Type of headache was classified according to the International Headache Society Criteria III $\beta$. Control group included age- and sex-matched relatives of patients attending medicine outpatient clinics.

Results: A total of 120 patients (69 males), their first-degree relatives, and equal number of controls were studied from June to December 2018. Mean age of the patients was $26.5 \pm 9.9$ years and mean epilepsy duration was $10.3 \pm 9.1$ years. Of these, 76 (63.3\%) had focal epilepsy and 40 (33.3\%) had idiopathic generalized epilepsy. In the epilepsy group, 72 (60\%) patients had interictal headache, 16 (13.3) had postictal headache, one $(0.8 \%)$ had ictal headache, while nine (7.5\%) patients had both interictal and postictal headaches. Interictal headache was present in 81 (67.5\%) patients $(25.8 \%$ migraine, $41.7 \%$ tension type headache [TTH]), 62 (51.6\%) relatives (24.2\% migraine and 27.5\% TTH), and 51 (42.5\%) controls (10.8\% migraine and $31.7 \% \mathrm{TTH})$. All types of headaches $(p=0.0002)$ and migraine $(p=0.004)$ were more common in patients than control wile migraine was also more common in relatives as compared with controls $(p=0.01)$.

Conclusion: Migraine is more common in patients with epilepsy and their first-degree relatives as compared with controls indicating a shared genetic link between epilepsy and migraine. 